\title{
Riesgo ergonómico y satisfacción laboral en trabajadores administrativos de la Municipalidad Distrital de Luyando en el periodo 2021
}

\section{Ergonomic risk and job satisfaction in administrative workers of the District Municipality of Luyando in the period 2021}

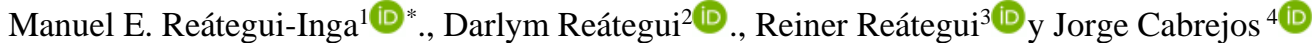

\begin{abstract}
RESUMEN
El objetivo del estudio fue determinar la relación entre el riesgo ergonómico y la satisfacción laboral en trabajadores administrativos de la Municipalidad Distrital de Luyando en el periodo 2021. El nivel del estudio fue correlacional de tipo transversal, la muestra estuvo conformada por el total de la población, 39 trabajadores administrativos. Para la recolección de datos se utilizó el Método RULA (Rapid Upper Limb Assessment) y la encuesta de satisfacción laboral. Los resultados mostraron correlación negativa débil entre el riesgo ergonómico y la satisfacción laboral; satisfacción laboral extrínseca (rho= -0.413 ; $\mathrm{p}=0.009<0.05)$ y satisfacción laboral intrínseca $(\mathrm{rho}=-0.305 ; \mathrm{p}=0.049<0.05)$; en tal sentido se concluye que a mayor riesgo ergonómico menor será la satisfacción laboral y viceversa.
\end{abstract}

Palabras clave: Ergonómico, Laboral, Satisfacción intrínsecos, Satisfacción extrínsecos.

\begin{abstract}
The objective of the study was to determine the relationship between ergonomic risk and job satisfaction in administrative workers of the District Municipality of Luyando in the period 2021. The level of the study was cross-sectional correlational, the sample consisted of the total population, 39 administrative workers. The RULA method (Rapid Upper Limb Assessment) and the job satisfaction survey were used for data collection. The results showed a weak negative correlation between ergonomic risk and job satisfaction; extrinsic job satisfaction $(\mathrm{rho}=-0.413$; $\mathrm{p}=0.009<0.05)$ and intrinsic job satisfaction $(\mathrm{rho}=$ $-0.305 ; \mathrm{p}=0.049<0.05$ ); in this sense it is concluded that the higher the ergonomic risk the lower the job satisfaction and vice versa.
\end{abstract}

Keywords: Ergonomic, Labor, Intrinsic Satisfaction, Extrinsic Satisfaction.

DOI: https://doi.org/10.37787/pakamuros-unj.v9i3.221

Recibido: 27/04/2020. Aceptado: 16/07/2021

* Autor para correspondencia

1. Universidad Nacional de Juliaca, Perú. Email: manuel.reategui@hotmail.com

2. Universidad Nacional Agraria de la Selva, Perú. Email: darlym.reategui@unas.edu.pe

3. Universidad Nacional Amazónica de Madre de Dios, Perú. Email: zootec2005@hotmail.com

4. Universidad Nacional de Jaén, Perú. Email: jorge_cabrejos@unj.edu.pe 


\section{INTRODUCCIÓN}

La ergonomía es un factor importante en las actividades laborales porque estudia el ambiente donde se desarrolla las actividades y como se adapta éste al trabajador (Prevalia, 2013). En el entorno donde se desenvuelve existe características que pueden generar una serie de trastornos o lesiones llamados riesgos ergonómicos. Los riesgos ergonómicos se manifiestan de diferentes maneras, desde la mala postura, esfuerzo físico, movimientos repetitivos y factores psicosociales que perjudican la satisfacción y rendimiento laboral (ErgoIBV, 2021).

A nivel internacional, en la segunda encuesta europea sobre riesgos nuevos y emergentes, donde España contó con una muestra de 3162 empresas encuestadas, encontraron factores de riesgo, tales como las posiciones que causan fatiga o dolor (79.2 \%) repeticiones intermitentes de manos y brazos (60.1 \%) (Sutil, 2021).

En el Perú, hay insuficientes reportes de riesgos en el trabajo de oficina, dificultando realizar el seguimiento y control del bienestar de los trabajadores, no obstante, lo que se aprecia son los diferentes casos reportados por instituciones públicas y privadas, de personal con lesiones que tienen limitaciones médicas en el trabajo. Wright y Davis (2003), menciona que la satisfacción laboral en los trabajadores es la sensación de bienestar al desempeño laboral. En ese sentido el Ministerio de Trabajo y Promoción del Empleo, para noviembre del 2008, promulgó la "Norma Básica de Ergonomía y de Procedimiento de Evaluación de Riesgo Disergonómico” Resolución Ministerial 375-2008-TR por exigencia del gobierno en la necesidad de adaptarse a las exigencias de los tratados de libre comercio. La aplicación en las instituciones públicas y privadas entra en auge a partir del año 2011 cuando se promulga la Ley de Seguridad y Salud en el Trabajo en donde ya se exige la realización de los análisis ergonómicos en las empresas (Ullilen, 2016).

Sobre la base de lo mencionado, el objetivo fue determinar la relación entre el riesgo ergonómico y la satisfacción laboral en trabajadores administrativos de la Municipalidad Distrital de Luyando en el periodo 2021.

\section{MATERIALES Y MÉTODOS}

El estudio fue de nivel correlacional y tipo transversal (Hernández et al., 2014). La muestra estuvo conformada por 39 trabajadores de las oficinas administrativas de la Municipalidad Distrital de Luyando. Los instrumentos para la recolección de datos fueron el método RULA y la encuesta de satisfacción laboral. 
El método RULA fue desarrollada por McAtamney y Corlett en 1993, divide a las partes del cuerpo en Grupo A (brazos, antebrazos y muñeca) y Grupo B (cuello, tronco y piernas) para evaluar el riesgo ergonómico, se aplicó el instrumento con validez y confiabilidad (García et al., 2013),

La encuesta de satisfacción laboral fue diseñada de acuerdo a Warr, Cook y Wall (1979) y se evaluó la escala de satisfacción laboral, tipo Likert consto de 15 ítems y fue distribuido en dos partes: satisfacción intrínseca (ítems pares) y extrínseca (ítems impares). Los valores fueron de 1 a 7 (1, 2, 3, 4, 5, 6 y 7) de acuerdo a su juicio del entrevistado (Tabla 1).

Tabla 1. Encuesta de satisfacción laboral

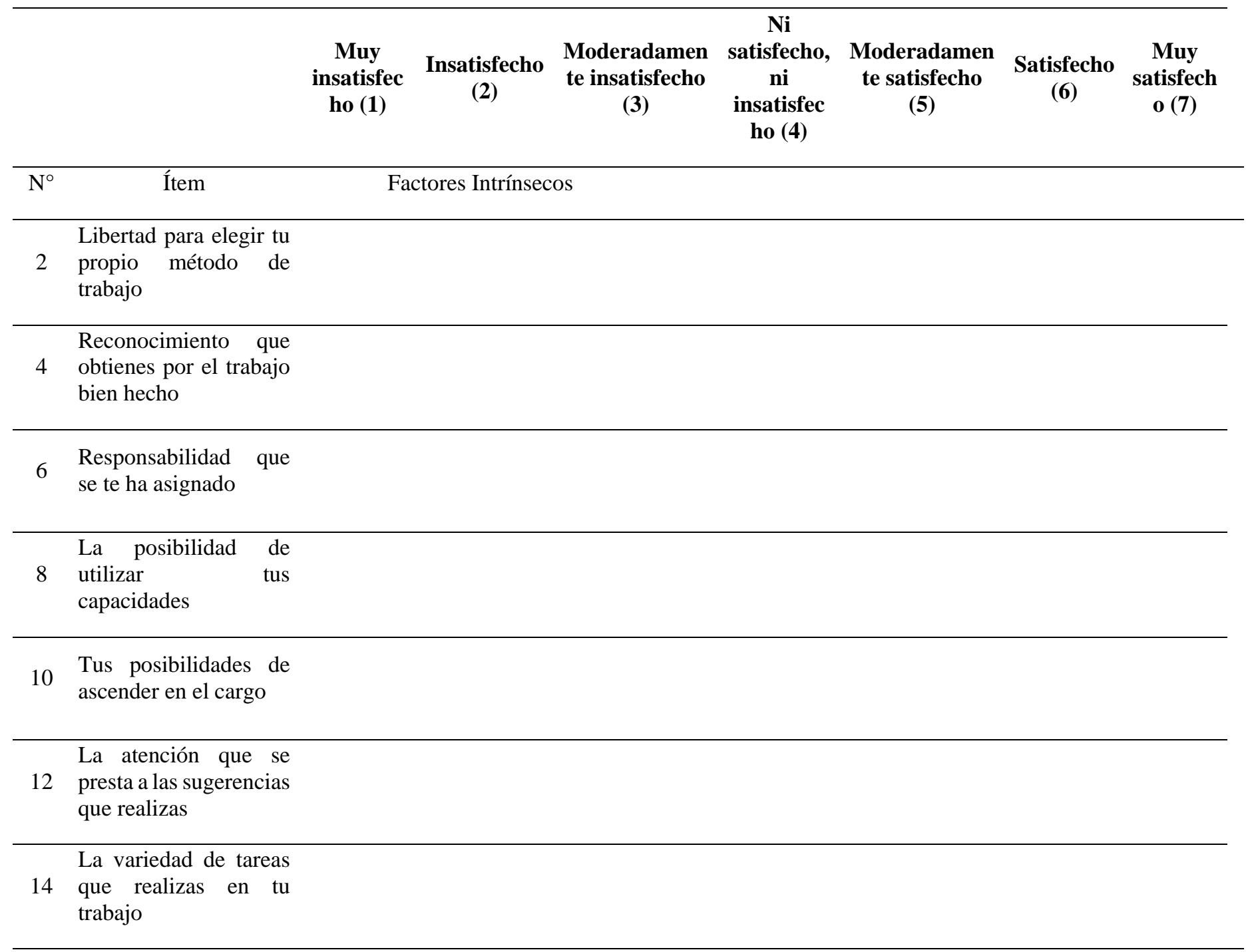

Factores Extrínsecos 
Condiciones físicas en

el trabajo

(iluminación, ruido,

espacio, etc.)

3 Tus compañeros de trabajo

5 Tu superior inmediato

7 Tu salario

\begin{tabular}{ll}
\hline $\begin{array}{l}\text { Relación entre tu } \\
\text { superior y los } \\
\text { trabajadores de tu área }\end{array}$ \\
\hline 11 & $\begin{array}{l}\text { El modo en que tu } \\
\text { institución } \\
\text { estructurada }\end{array}$ \\
\hline
\end{tabular}

13 Tu horario de trabajo

15 Tu estabilidad laboral

Fuente: Adaptado de Warr et al. (1979)

La encuesta fue validada por 5 expertos, el valor del coeficiente de validez de contenido fue de 0.91 (extrínseca) y 0.93 (intrínseca), que indica una muy buena validez y con respecto a la confiabilidad se utilizó el estadígrafo Alfa de Cronbach, el cual arrojó un valor de 0.91 indicando una excelente confiabilidad (Tuapanta et al., 2017).

Para la ejecución del estudio se solicitó los permisos correspondientes a las autoridades de la Municipalidad, una vez obtenida el consentimiento, se procedió a la evaluación in situ en las oficinas que consistió en la toma de fotografías (lateral, trasera y superior) de las posturas de los trabajadores mientras hacían uso de la computadora o laptop. Posteriormente, para medir los ángulos formados por las partes del cuerpo se utilizó el software online RULER (Ergonautas, 2021a) y seguidamente asignar puntuaciones de acuerdo al método RULA y así determinar el nivel de actuación de la tarea por trabajador (Ergonautas, 2021b).

Para efectuar el proceso de los datos en el riesgo ergonómico y la encuesta de satisfacción laboral se utilizó el programa Microsoft Excel 2016. Así mismo se determinó la confiabilidad y normalidad de los 
datos mediante los estadígrafos Alfa de Cronbach y Kolmogorov-Smirnov. La correlación de las variables se determinó aplicando el estadígrafo Rho Spearman utilizando el Software SPSS 26.

\section{RESULTADOS}

Figura 1, se observa el nivel de actuación de la tarea promedio, donde el mayor porcentaje fue para el nivel 2 y 3 con 61.54 y $30.77 \%$ respectivamente.

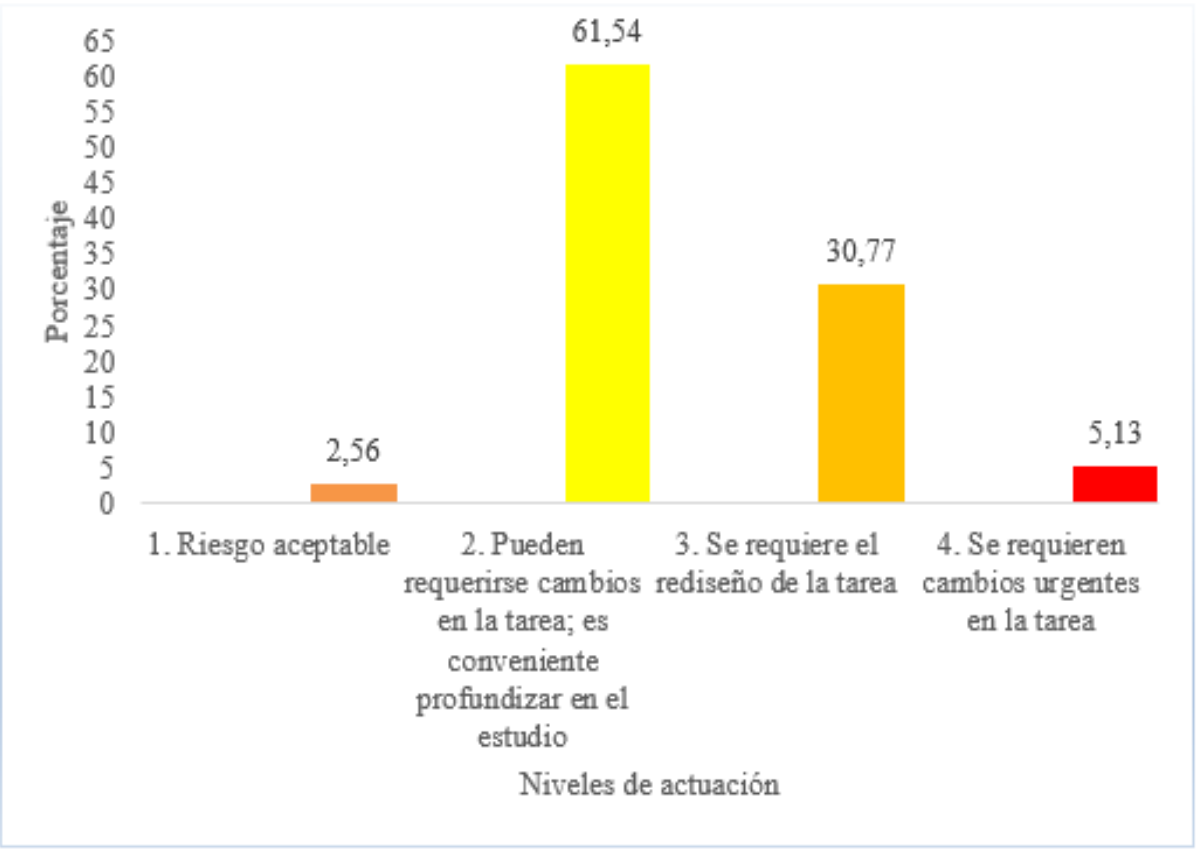

Figura 1. Nivel de actuación de los trabajadores administrativos de la Municipalidad Distrital de Luyando

En lo referente a la encuesta de satisfacción laboral intrínseca promedio, se aprecia la escala 5 moderadamente satisfecho, que corresponde al ítem de aspiraciones a ascender en sus cargos. Mientras que los ítems 6 estuvo en la escala satisfecho (Figura 2). 


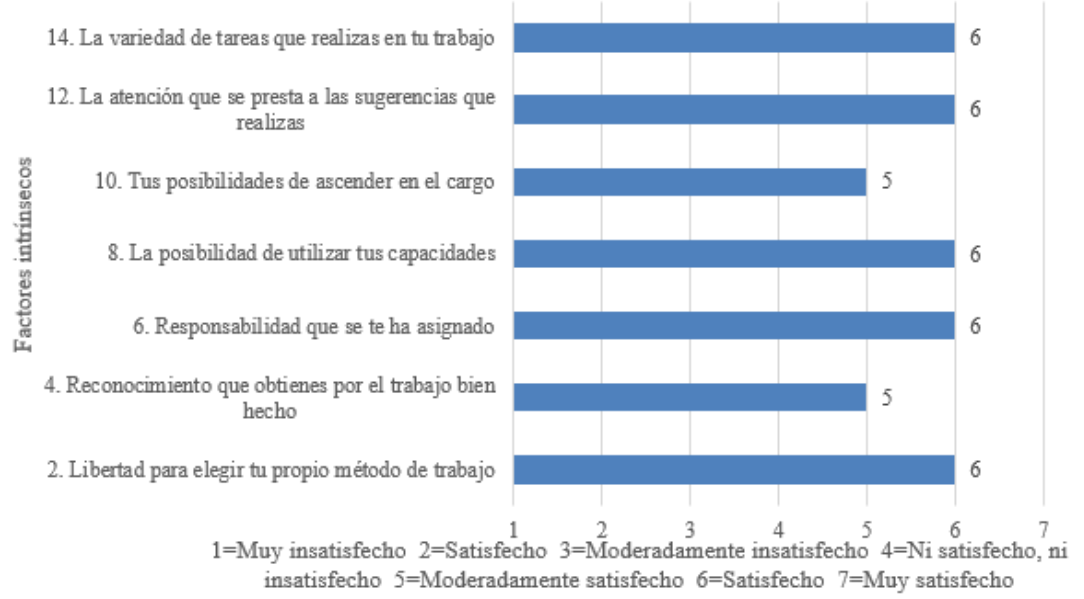

Figura 2. Encuesta de satisfacción laboral promedio de los factores intrínsecos.

Con respecto a la encuesta de satisfacción laboral extrínseca promedio, se muestra la escala 5 (Moderadamente satisfecho) para los ítems 1, 7 y 11, y escala 6 (Satisfecho) (Figura 3).

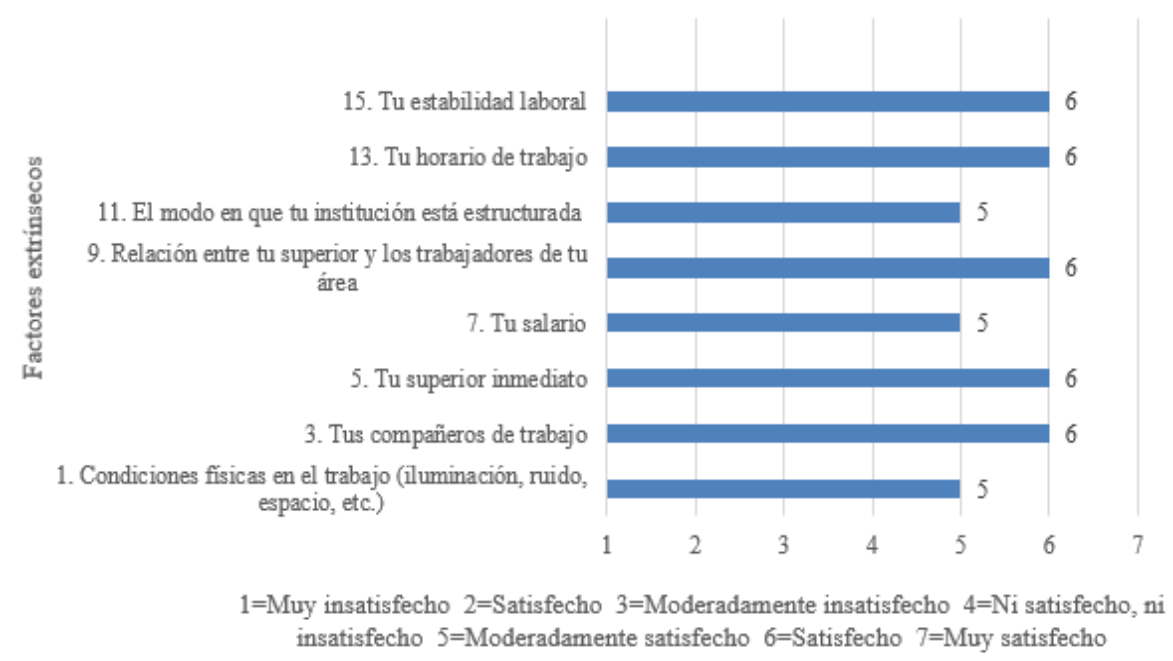

Figura 3. Encuesta de satisfacción laboral promedio de los factores extrínsecos.

En lo que concierne a la correlación de las variables de estudio en la Tabla 2, se observa que existe correlación negativa débil entre el riesgo ergonómico y la satisfacción laboral; satisfacción laboral extrínseca (rho= -0.413; $\mathrm{p}=0.009<0.05)$ y satisfacción laboral intrínseca $(r h o=-0.305 ; \mathrm{p}=0.049<0.05$ ); el cual indica que a mayor riesgo ergonómico menor será la satisfacción laboral. 
Tabla 2. Correlación Rho de Spearman de las variables riesgo ergonómico y satisfacción laboral intrínseca y extrínseca

\begin{tabular}{|c|c|c|c|}
\hline \multicolumn{4}{|c|}{ Riesgo ergonómico y satisfacción laboral intrínseca } \\
\hline Variable & Estadístico & $\begin{array}{c}\text { Riesgo } \\
\text { ergonómico }\end{array}$ & $\begin{array}{c}\text { Satisfacción } \\
\text { laboral } \\
\text { intrínseca }\end{array}$ \\
\hline \multirow{3}{*}{ Riesgo ergonómico } & $\begin{array}{l}\text { Coeficiente de } \\
\text { correlación }\end{array}$ & 1.000 & -0.413 \\
\hline & Sig. (bilateral) & . & 0.009 \\
\hline & $\mathrm{N}$ & 39 & 39 \\
\hline \multirow{3}{*}{ Satisfacción laboral intrínseca } & $\begin{array}{l}\text { Coeficiente de } \\
\text { correlación }\end{array}$ & -0.413 & 1.000 \\
\hline & Sig. (bilateral) & 0.009 & . \\
\hline & $\mathrm{N}$ & 39 & 39 \\
\hline \multicolumn{4}{|c|}{ Riesgo ergonómico y satisfacción laboral extrínseca. } \\
\hline Variable & Estadístico & $\begin{array}{l}\text { Riesgo } \\
\text { ergonómico }\end{array}$ & $\begin{array}{c}\text { Satisfacción } \\
\text { laboral } \\
\text { extrínseca }\end{array}$ \\
\hline \multirow{3}{*}{ Riesgo ergonómico } & $\begin{array}{l}\text { Coeficiente de } \\
\text { correlación }\end{array}$ & 1.000 & -0.305 \\
\hline & Sig. (bilateral) & . & 0.049 \\
\hline & $\mathrm{N}$ & 39 & 39 \\
\hline \multirow{3}{*}{ Satisfacción laboral extrínseca } & $\begin{array}{l}\text { Coeficiente de } \\
\text { correlación }\end{array}$ & -0.305 & 1.000 \\
\hline & Sig. (bilateral) & 0.049 & . \\
\hline & $\mathrm{N}$ & 39 & 39 \\
\hline
\end{tabular}

\section{DISCUSIÓN}

El nivel de actuación de la tarea de los trabajadores, se presenta porcentajes mayores en el nivel 2 y 3, debiéndose tal vez a que la ergonomía no es un tema de prioridad en la institución por no contar con un plan y estrategias ergonómicas (Aravena y Pino, 2010). Por otro lado, al no realizarse estos cambios en las tareas podrían traer consecuencias a la salud a largo plazo en los trabajadores, en donde se ha comprobado que el papel del trabajador en materia ocupacional está vinculado con el riesgo físico, dado que requiere de largos periodos en posición sentada, involucrando los músculos de la espalda, hombros incluido el cuello, las repeticiones en los movimientos del hombro y muñeca, que incrementa el riesgo de aparición de síntomas dolorosos (Vernaza y Sierra, 2005; Vieira y Kumar, 2004). La prevalencia de la posición sentada, se vincula con la presencia de síntomas musculoesqueléticos en la parte superior, espalda baja y parte inferior del cuerpo (Coenen et al., 2018). Los trabajadores al realizar sus actividades frente al ordenador, presentan escaza actividad física y como consecuencia tienen un índice elevado de sedentarismo, sobrepeso u obesidad, incrementando el nivel de fatiga muscular y dolor (Merlino et al., 
2003; Lebouf, 2000); comprometiendo negativamente las actividades laborales y extralaborales (Sauter y Moon, 1996; Loghmani et al., 2013).

Young (2015) menciona que la satisfacción laboral está determinada cuando la gerencia mayor destina monetariamente en el desarrollo de las políticas de la organización, en este contexto, se puede suponer que la Municipalidad Distrital de Luyando invierte en sus políticas organizacionales; ya que, de los 15 ítems, en 10 ítems los trabajadores indican que se sienten Satisfechos y en el resto Moderadamente satisfechos.

En los factores intrínsecos y extrínsecos Murruga (2016) consideró para el primero: autonomía en el trabajo, diversidad de actividades, pertinencia de promoción en las actividades, gratitud por la actividad realizada y en lo segundo: retribución económica, ambiente físico, seguridad laboral, dirección institucional, charlas y capacitaciones; los trabajadores afirmaron que tienen un elevado nivel de satisfacción laboral, lo que coincide con los resultados hallados los cuales revelan que los trabajadores en promedio se sienten entre Moderadamente satisfechos y Satisfechos.

En cuanto a los factores psicosociales, Gil (2009) indica que son condiciones presentes relacionadas con el puesto, el tipo de trabajo en la ejecución de la actividad e incluso con el ambiente. Jurado (2017) en su investigación menciona que mientras mejor sea el entorno laboral, el indicador de satisfacción será elevado dentro de las instituciones evaluadas; del mismo modo los factores psicosociales se pueden asociar con los factores intrínsecos que también se refiere al contenido, tarea y deberes relacionados con el puesto laboral (Herzberg, 1989). En consecuencia, se podría afirmar que los trabajadores cuentan con factores psicosociales positivos lo cual se ve reflejado en los promedios de satisfacción laboral que oscilan entre moderadamente satisfecho y satisfecho.

Justiniano (2017) en trabajadores del SENASA, indica que el entorno físico, comodidad, confortable relación con su superior de área, el horario de trabajo, la remuneración y los beneficios laborales, incrementan el nivel de satisfacción laboral, estas características mencionadas coinciden con los ítems 1 (condiciones físicas en el trabajo), 5 (tu superior inmediato), 13 (tu horario de trabajo), 7 (tu salario) y 10 (tus posibilidades de ascender en el cargo) en donde los trabajadores administrativos mencionan que se sienten entre moderadamente satisfechos y satisfechos.

Con respecto al ítem 4 (reconocimiento que obtienes por el trabajo bien hecho) se tiene escala de satisfacción laboral de 5 (Moderadamente satisfecho), se supone que incentivando y reconociendo su trabajo dicha escala puede elevarse a 7 (Muy satisfecho), como lo indica Ramos (2019) en su 
investigación, la mayoría de los trabajadores al recibir un incentivo y/o reconocimiento están satisfechos en su trabajo y ello es una forma de motivarlos para lograr las metas de la institución.

A partir de las hipótesis comprobadas, en donde los valores Rho de Spearman indican una correlación negativa débil entre el riesgo ergonómico y la satisfacción laboral intrínseca y extrínseca, de forma similar, las hipótesis comprobadas por Marroquín (2017) también presentan el mismo tipo de correlación.

\section{CONCLUSIONES}

Los trabajadores no cuentan con mobiliario ergonómico ni con capacitaciones en posturas ergonómicas lo cual se vio evidenciado en los resultados, de continuar así, corren el riesgo a largo plazo de sufrir problemas de salud como los trastornos musculoesqueléticos, para evitar esa situación se sugiere a la Municipalidad crear el Comité de Seguridad y Salud en el Trabajo que se encargará de realizar los planes y programas en buenas prácticas posturales en el trabajo.

\section{REFERENCIAS BIBLIOGRÁFICAS}

Aravena, M. y Pino, C. (2010). Ergonomía: Impacto en la Productividad y Satisfacción en los Trabajadores de Empresas Industriales en la Ciudad de Valdivia [Tesis de pregrado, Universidad Austral de Chile]. Archivo digital. http://cybertesis.uach.cl/tesis/uach/2010/fea663e/doc/fea663e.pdf

Coenen, P., Healy, G., Winkler, E., Dunstan, D., Owen, N., Moodie, M., LaMontagne, A., Eakin, E., O'Sullivan, P. y Straker, L. (2018). Associations of office worker's objectively assessed occupational sitting, standing and stepping time with musculoskeletal symptoms. Ergonomics, 61(9), 1187-1195. https://doi.org/10.1080/00140139.2018.1462891

ErgolBV. (2021). Riesgos ergonómicos: medidas para prevenirlos. http://www.ergoibv.com/blog/riesgos-ergonomicos-medidas-para-prevenirlos/

Ergonautas. (2021a). RULER - Medición de ángulos en fotografía. https://www.ergonautas.upv.es/herramientas/ruler/ruler.php Ergonautas. (2021b). RULA. https://www.ergonautas.upv.es/metodos/rula/rula-ayuda.php 
García, M., Sánchez, A., Camacho, A. y Domingo, R. (2013). Análisis de métodos de valoración postural en las herramientas de simulación virtual para la ingeniería de fabricación. Dyna, 80, 5-15. http://www.redalyc.org/pdf/3604/360433575004.pdf.

Gil, P. (2009). Algunas razones para considerar los riesgos psicosociales en el trabajo y sus consecuencias en la salud pública. Revista Española de Salud Pública, 83(2), 169-173. https://www.scielosp.org/article/resp/2009.v83n2/169-173/es/

Hernández, R., Fernández, C. y Baptista, P. (2014). Metodología de la investigación científica. McGraw McGraw Hill. https://www.uca.ac.cr/wp-content/uploads/2017/10/Investigacion.pdf

Herzberg, F. (1989). Teoría de la Higiene. México. Editorial Mc Graw-Hill.

Jurado, P. (2017). Los factores psicosociales y la satisfacción laboral en Gobiernos Autónomos Descentralizados Municipales de la provincia de Tungurahua [Tesis de maestría, Universidad Técnica de Ambato] Repositorio digital. https://repositorio.uta.edu.ec/jspui/handle/123456789/32059

Justiniano, E. (2017). Ergonomía y satisfacción laboral en los servidores del Servicio Nacional de Sanidad Agraria, Lima 2017 [Tesis de maestría, Universidad César Vallejo]. Repositorio digital. https://hdl.handle.net/20.500.12692/16072

Lebouf, C. (2000). Body weight and low back pain. Spine, 25, 226-237. https://doi.org/10.1097/00007632-200001150-00015

Loghmani, A., Golshiri, P., Zamani, A., Kheirmand, M. y Jafari N. (2013). Musculoskeletal symptoms and job satisfaction among office-workers: a cross-sectional study from Iran. Acta Médica Académica, 42(1), 46-54. https://doi.org/10.5644/ama2006-124.70

Marroquín, J. (2017). Riesgo ergonómico laboral en trabajadores administrativos de un instituto especializado de salud [Tesis de maestría, Universidad Cesar Vallejo] Repositorio institucional. http://repositorio.ucv.edu.pe/handle/20.500.12692/8781

McAtamney, L. y Corlett, E. (1993). RULA: a survey method for the investigation of work-related upper limb disorders. Applied Ergonomics, 24(2), 91-99. https://doi.org/10.1016/0003-6870(93)90080$\mathrm{S}$

Merlino, L., Rosecrance, J., Anton D. y Cook, T. (2003). Symptoms of musculoskeletal disorders among apprentice construction workers. Applied Occupational and Environmental Hygiene, 18, 67-72. https://doi.org/10.1080/10473220301391 
Murrugarra, J. (2016). La ergonomía y satisfacción laboral de los trabajadores de la municipalidad distrital de Pachacamac en el periodo 2016 [Tesis de maestría, Universidad Cesar Vallejo] $\begin{array}{llll}\text { Repositorio de la Universidad } & \text { César }\end{array}$ http://repositorio.ucv.edu.pe/handle/20.500.12692/6682

Prevalia, S. (2013). Riesgos ergonómicos y medidas preventivas. http://www.ajemadrid.es/wpcontent/uploads/aje_ergonomicos.pdf.

Ramos, D. (2019). La motivación y el desempeño laboral de los trabajadores del restaurant Sol de Mayo SCRL Huánuco 2019 [Tesis de grado, Universidad de Huánuco] Repositorio institucional. http://repositorio.udh.edu.pe/123456789/2252

Sauter, S. y Moon, S.D. (Eds.). (1996). Beyond Biomechanics: Psychosocial Aspects Of Musculoskeletal Disorders In Office Work (1st ed.). CRC Press. https://doi.org/10.1201/9781482272680

Sutil, J. (2021). Factores de riesgo psicosocial en España. https://affor.es/factores-de-riesgo-psicosocialen-espana/

Tuapanta J., Duque, M. y Mena, R. (2017). Alfa de Cronbach para validar un cuestionario de uso de TIC en docentes universitarios. Revista mktDescubre, https://core.ac.uk/download/pdf/234578641.pdf

Vernaza, P. y Sierra, C. (2005). Dolor muscuesquelético y su asociación con factores de riesgos ergonómicos, en trabajadores administrativos. Revista de salud pública, 7(3), 317-326. http://www.scielo.org.co/scielo.php?script=sci_abstract\&pid=S0124-00642005000300007

Ullilen, C. (2016). La aplicación de la ergonomía en el Perú. https://www.ergonomaullilen.com/blog/laaplicacion-de-la-ergonomia-en-el-peru/84/

Vieira, E. y Kumar, S. (2004). Working postures: a literature review. Journal of Occupational Rehabilitation, 14(2), 143-59. https://www.ncbi.nlm.nih.gov/pubmed/15074366

Warr P, Cook J, Wall T. (1979). Scales for the measurement of some work attitudes and aspects of psychological well-being. Journal of Occupational Psychology, 52, 129-148. https://doi.org/10.1111/j.2044-8325.1979.tb00448.x

Wright, B. y Davis, B. (2003). Job Satisfaction In The Public Sector: The Role of the Work Environment. The American Review of Public Administration, 33(1), 70-90. https://doi.org/10.1177/0275074002250254

Young, L. (2015). Comparison of Job Satisfaction Between Nonprofit and Public Employees. SAGE journals, 45(2), 295-313. https://doi.org/10.1177/0899764015584061 